\title{
The Drift Velocity of the Geomagnetic Secular Variation
}

\author{
Takesi Yukutake \\ Earthquake Research Institute, University of Tokyo \\ (Received August 1, 1968)
}

\begin{abstract}
Based on the spherical harmonic analyses of the geomagnetic secular variation, the drift velocity of the earth's magnetic field is examined in three ways. In the first place, drift rates of phase angles of individual spherical harmonic components are calculated. Secondly the geomagnetic secular variations along parallel circles are expanded in Fourier series and drift velocities of harmonic components for various latitudes are estimated. Thirdly a rigid rotation is assumed for the westward drift of the secular variation along a parallel and the velocity is determined by calculating a correlation function between the distributions of the secular change at different epochs.

From these it has resulted in that there exists a dispersive relationship in the drift rate, at least between the harmonic component $m=1$ and $m=2$. The velocity of the westward drift is independent of the latitude except for a narrow zone near the equator, where an occasional eastward drift is observable.

It has been confirmed that the mean drift rate of the secular variation $\left(0.3^{\circ} /\right.$ year for a rigid rotation model) is definitely larger than that of the non-dipole field itself $\left(0.2^{\circ} /\right.$ year $)$. The low mean velocity of the non-dipole field seems to arise from the coexistence of the stationary fields with the drifting ones.
\end{abstract}

\section{Introduction}

The westward drift of the earth's magnetic field has long been noticed since about the 17 th century. At present it is one of the most firmly established phenomena in geomagnetism. With regard to the velocity of the drift, however, there are many discussions. As the methods and techniques of the analyses vary, the diverse velocities are obtained, and there are still many things left uncertain about the nature of the drift.

One of the problems about the drift velocity is whether any dispersive relationship exists in the drift velocities or not. Time variation in the harmonic constituents of the earth's magnetic field was examined by several authors. The results indicate that the individual phase angles of the harmonic components are likely to have shifted their longitudes with different velocities (Bulla.d et al., 1950; Whitham, 1958; Nagata, 1962; Adam et al., 1964 ; Hide, 1966, p. 640), but they are not conclusive enough to reach a quantitative agreement. The second problem is if there is any latitude dependence of the drift velocities. For recent data, the drift velocities are low near $40^{\circ} \mathrm{N}$ and higher in the low latitudes (Bullard et al., 1950; Yukutake, 1962; Adam et al., 1964), whilst an analysis for historic data suggests an increase in the velocity with the latitude (Yukutake, 1967).

Another problem is the discrepancy between the velocities obtained from the non-dipole 
field and those from the geomagnetic secular variation. Specific features of the non-dipole field seem to drift westwards with their own velocities, but when an average all over the world is taken, the westward velocity is approximately $0.2^{\circ} /$ year (Bullard et al., 1950; Yukutake, 1962; Nagata, 1962; Adam et al., 1964). On the other hand, comparison of isoporic charts for different epochs gives the mean rate of drift $0.32^{\circ} /$ year (Bullard et al., 1950). The difference between these two estimates seems significant certainly exceeding possible errors involved in the analyses of the geomagnetic field.

It was recently pointed out by Yukutake and Tachinaka (1968a) that, among the nondipole regional anomalies, there were a number of anomalies standing at the same position over a few hundred years, and that only a few had drifted westwards. However, examination of isoporic charts for different epochs revealed that the whole distribution of the geomagnetic secular variation had been drifting irrespective of the standing non-dipole anomalies, and confirmed that westward drift was a worldwide phenomenon rather than a local one restricted only to a few drifting non-dipole anomalies (Yukutake and Tachinaka, 1968b).

The standing non-dipole anomalies probably reduce the mean drift rate of the nondipole field when they are included in the calculation of the world average. On the other hand, the migration of the isopors is supposedly less influenced by the standing anomalies, unless these anomalies change their intensities markedly. Therefore, the volocity obtained from the secular variation seems to give a better approximation as that of the drifting anomaly.

In this paper, three different methods are presented for calculating the drift velocities from the spherical harmonic coefficients of the geomagnetic secular variation. In the first place the drift rates are computed for individual harmonic components. Secondly the geomagnetic secular variation is expanded in a Fourier series along a parallel circle and the drift velocities for each wave number are calculated. In the last place, correlating the secular variation at a certain epoch to those at different epochs, the mean drift velocity during the period is estimated.

\section{The westward drift of the harmonic constituent of the geomagnetic secular variation}

In a previous paper, going back to $1743 \mathrm{AD}$, spherical harmonic coefficients of the geomagnetic secular variation were computed from the change in the coefficients of the main field (Yukutake and Tachinaka, $1968 \mathrm{~b}$ ). Employing these, we can examine the time variations in the phase angles of the harmonic constituents. Let $\dot{g}_{n}{ }^{m}$ and $\dot{h}_{n}{ }^{m}$ be the coefficients for cosine and sine terms of degree $n$ and order $m$. Then the magnetic potential $V$ for the secular change can be written as follows,

$$
\left.\begin{array}{c}
V=a \sum_{n=1}^{\infty} \sum_{m=0}^{n}\left\{\dot{g}_{n}{ }^{m} \cos m \phi+\dot{h}_{n}{ }^{m} \sin m \phi\right\} P_{n}{ }^{m}(\cos \theta) \\
=a \sum_{n=1}^{\infty} \sum_{m=0}^{n} \dot{C}_{n}{ }^{m} \cos m\left(\phi-{\phi_{n}}^{m}\right) P_{n}{ }^{m}(\cos \theta), \\
\phi_{n}{ }^{m}=\frac{1}{m} \tan ^{-1} \frac{\dot{h}_{n}{ }^{m}}{\dot{g}_{n}{ }^{m}}, \\
\dot{C}_{n}{ }^{m}=\sqrt{{\dot{g_{n}}}^{m 2}+\dot{h}_{n}{ }^{m 2}},
\end{array}\right\}
$$




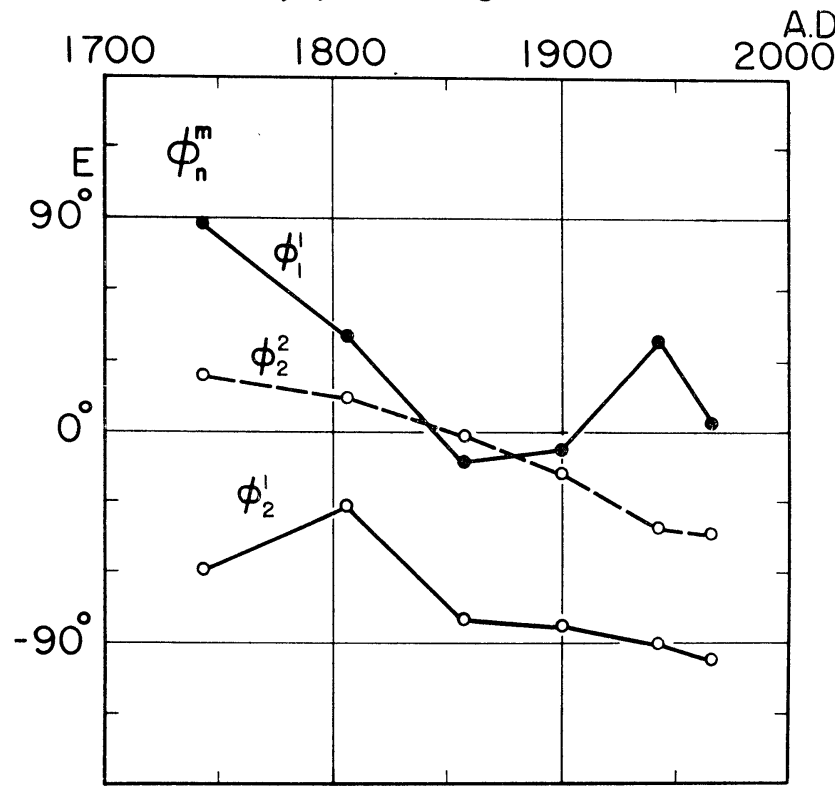

Fig. 1 Time variations in phase angles of the spherical harmonic components of the secular change potential, $\phi_{1}{ }^{1}, \phi_{2}{ }^{1}$ and $\phi_{2}{ }^{2}$. Decrease in $\phi_{: 2}{ }^{m}$ indicates a westward drift of each component. Drift velocities obtained from the slopes are listed in Table 1 together with the standard deviations of individual values.

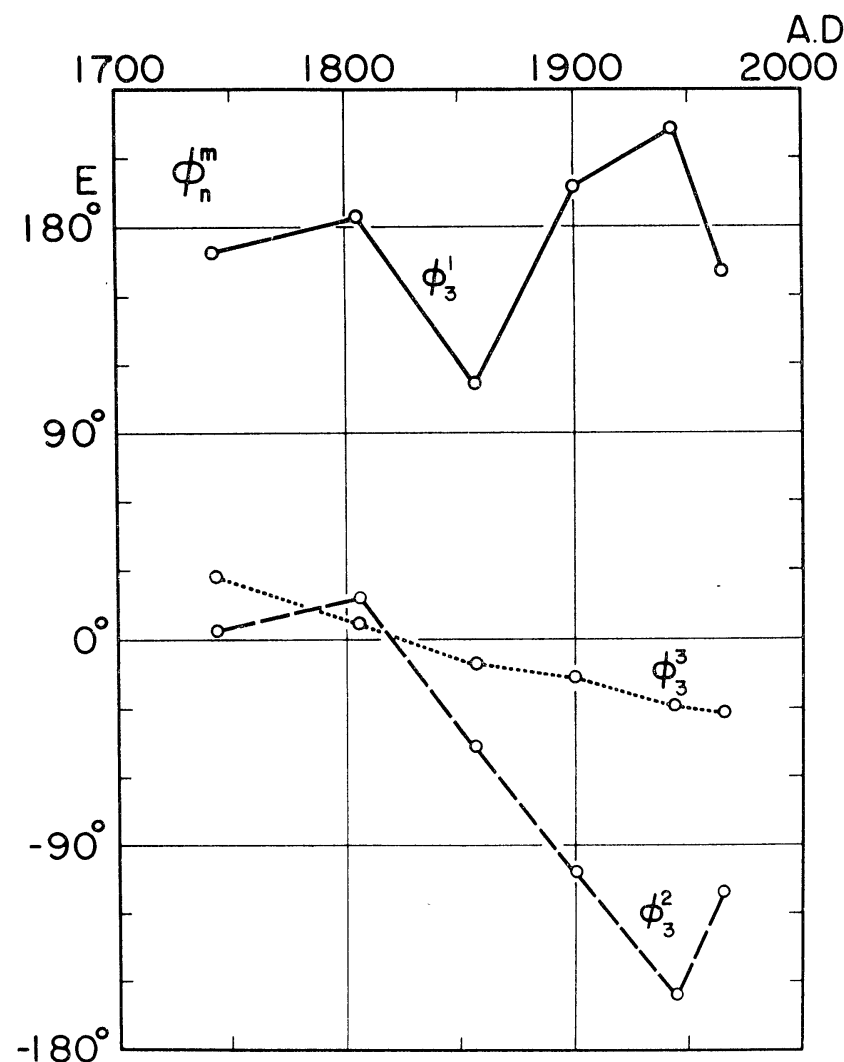

Fig. 2 Time variation in phase angles of the spherical harmonic components of the secular change potential, $\phi_{3}{ }^{1}, \phi_{3}{ }^{2}$ and $\phi_{3}{ }^{3}$. 
where $\theta$ and $\phi$ denote the colatitude and the east longitude. $P_{n}{ }^{m}(\cos \theta)$ is a Schmidt's halfnormalized spherical function and $a$ is the mean radius of the earth. Then the westward drift of the harmonic constituent can be shown by a decrease of $\phi_{n}{ }^{m}$ with time.

$\phi_{n}{ }^{m}$ was calculated for the secular change of 1743, 1806, 1857, 1900, 1942.5 and 1965 AD (see Yukutake and Tachinaka, $1968 \mathrm{~b}$ ) up to $n=4, m=4$ and shown in Figs. 1 and 2. Linear decrease is clearly noted with $\phi_{2}{ }^{2}$ and $\phi_{3}{ }^{3}$. Although the values are more scattered, $\phi_{1}{ }^{1}, \phi_{2}{ }^{1}$ and $\phi_{3}{ }^{2}$ decrease with time as well. As for $\phi_{3}{ }^{1}$, however, we can notice rather increase in phase with time, indicating an eastward drift, though each value scatters very widely. It will be discussed in some detail in a later paper whether the scatter of $\phi_{3}{ }^{1}$ is entirely due to the errors involved in the coefficients or that it has certain significance. For the $\phi_{4}{ }^{m}$ 's of degree 4 , systematic variation was not clearly seen,

Straight lines were fitted to each $\phi_{n}{ }^{m}$ by the least squares method. The gradients of the lines give the drift velocities for individual harmonics. They are listed in Table 1 together with the standard deviations, where the westward motion is taken positive. It is interesting to note that the sectorial harmonics drift westwards very steadily. The mean velocities of $\phi_{2}{ }^{2}$ and $\phi_{3}{ }^{3}$ are $0.33^{\circ} /$ year and $0.27^{\circ} /$ year respectively, and the standard deviations are $0.03^{\circ} /$ year and $0.02^{\circ}$ year. Next to these, $\phi_{2}{ }^{1}$ shows a linear westward movement with a velocity of $0.23^{\circ} /$ year and a standard deviation $0.09^{\circ} /$ year. As will be discussed later, an eastward motion of $\phi_{3}{ }^{1}$ seems to be associated with a counter drift of the secular variation in a narrow region near the equator.

Table 1. Drift velocities of the harmonic components of the geomagnetic secular variation.

\begin{tabular}{cc|c|c}
\hline$n$ & $m$ & Velocity $^{1)}(\% /$ year $)$ & $\sigma_{v}^{2)}(\% /$ year $)$ \\
\hline 1 & 1 & 0.297 & 0.173 \\
2 & 1 & 0.229 & 0.087 \\
2 & 2 & 0.334 & 0.025 \\
3 & 1 & -0.109 & 0.220 \\
3 & 2 & 0.733 & 0.168 \\
3 & 3 & 0.267 & 0.018 \\
\hline
\end{tabular}

1) Velocity is taken positive for the westward drift

2) Standard deviation of the velocity

\section{The westward drift of the Fourier components along parallel circles}

When a parallel circle is specified, distribution of the secular change along the circle can be expressed in terms of Fourier series. From equation (1), the magnetic potential $V$ is written as follows,

$$
V=a \sum_{m=0}^{\infty}\left\{\dot{G}_{m} \cos m \phi+\dot{H}_{m} \sin m \phi\right\}
$$

where

$$
\begin{aligned}
& \dot{G}_{m}=\sum_{n=m}^{\infty} \dot{g}_{n}{ }^{m} P_{n}{ }^{m}(\cos \theta), \\
& \dot{H}_{m}=\sum_{n=m}^{\infty} \dot{h}_{n}{ }^{m} P_{n}{ }^{m}(\cos \theta) .
\end{aligned}
$$




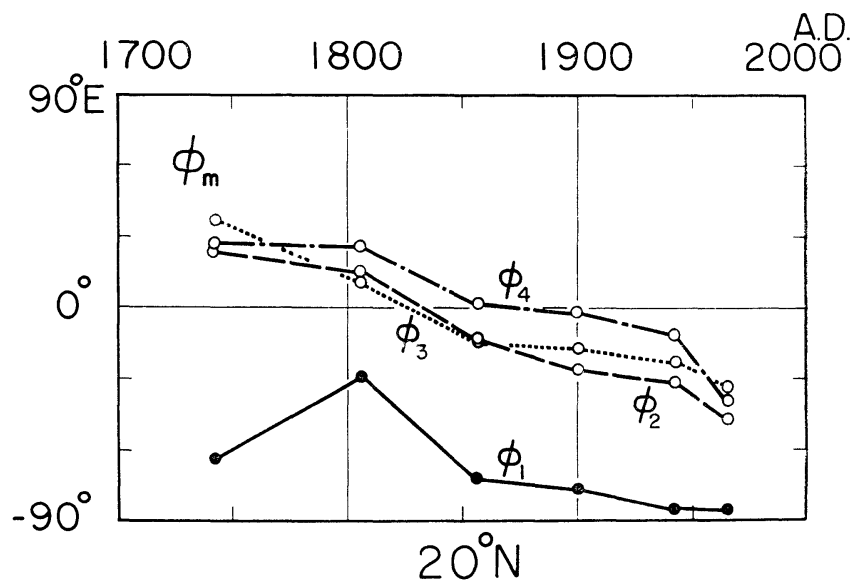

Fig. 3 Westward drift of Fourier components of the secular change potential along $20^{\circ} \mathrm{N}$ parallel circle.
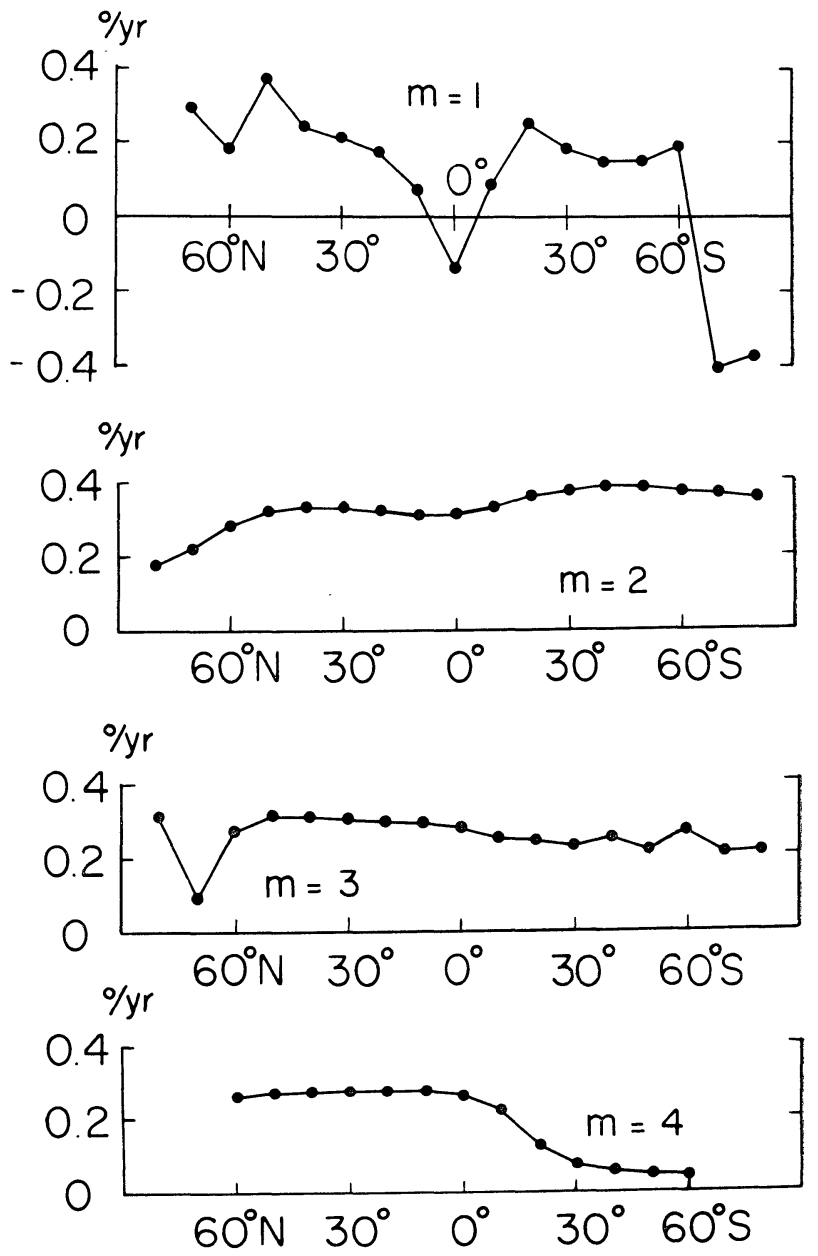

Fig. 4 Drift velocities of Fourier components of the secular change potential for various latitudes. The westward drift is taken positive. 
Similar to equation (2), we define $\phi_{m}$ by

$$
\phi_{m}=\frac{1}{m} \tan ^{-1} \frac{\dot{H}_{m}}{\dot{G}_{m}} .
$$

Time variation in $\phi_{m}$ gives the movement of Fourier component along the parallel circle.

Based on the same data as in the previous section, $\phi_{m}$ 's were calculated up to $m=4$ for various epochs from $80^{\circ} \mathrm{N}$ to $80^{\circ} \mathrm{S}$. An example is shown in Fig. 3 for $20^{\circ} \mathrm{N}$. A linear decrease with time is seen for all the $\phi_{m}$ 's. Straight lines were fitted by the least squares method and drift velocities were obtained from their slopes. The results are shown in Fig. 4 and Table 2.

Drift velocities for $\phi_{1}$ are fairly scattered especially for higher latitudes. Since the data of earlier analyses are dubious for higher latitudes, we shall discard the results for the latitudes higher than $60^{\circ}$. Then most of the velocities in middle latitudes range from $0.15^{\circ} /$ year to $0.25^{\circ}$ year. In low latitudes the velocity becomes small. At the equator an eastward velocity amounting to $0.14^{\circ}$ year was obtained. This counter movement is consistent with the eastward drift of the harmonic $n=3, m=1$ obtained in the previous section. A similar eastward drift was also observed for the harmonic $m=1$ of the non-dipole field itself.

Correlating the observed secular change with the eastward gradient of the field, we can calculate the drift velocity in the east-west direction. Fig. 5 was thus obtained for $m=1$

Table 2. Drift velocities of Fourier components of the geomagnetic secular variation

\begin{tabular}{|c|c|c|c|c|c|c|c|c|}
\hline \multirow{2}{*}{ Latitude } & \multicolumn{2}{|c|}{$m=1$} & \multicolumn{2}{|c|}{$m=2$} & \multicolumn{2}{|c|}{$m=3$} & \multicolumn{2}{|c|}{$m=4$} \\
\hline & $\begin{array}{c}\text { Velocity }{ }^{1)} \\
\left(0 / y^{\prime} .\right)\end{array}$ & $\begin{array}{c}\sigma_{v}^{2)} \\
\left({ }^{\circ} / \mathrm{yr} .\right)\end{array}$ & $\begin{array}{c}\text { Velocity }^{1)} \\
(0 / \mathrm{yr} .)\end{array}$ & $\begin{array}{c}\sigma_{v}{ }^{2)} \\
\left({ }^{\circ} / \mathrm{yr} .\right)\end{array}$ & $\begin{array}{l}\text { Velocity }{ }^{1)} \\
(0 / \text { yr. })\end{array}$ & $\begin{array}{c}\sigma_{v}^{2)} \\
(\% / y r .)\end{array}$ & $\begin{array}{c}\text { Velocity }^{1)} \\
\left({ }^{\circ} / \text { yr. }\right)\end{array}$ & $\begin{array}{c}\sigma_{v}{ }^{2)} \\
(\% / \text { yr. })\end{array}$ \\
\hline $80^{\circ} \mathrm{N}$ & 0.945 & 0.721 & 0.178 & 0.060 & 0.313 & 0.150 & & \\
\hline $70^{\circ}$ & 0.299 & 0.705 & 0.218 & 0.138 & 0.096 & 0.701 & & \\
\hline $60^{\circ}$ & 0.189 & 0.210 & 0.285 & 0.029 & 0.270 & 0.150 & 0.259 & 0.083 \\
\hline $50^{\circ}$ & 0.376 & 0.144 & 0.324 & 0.030 & 0.309 & 0.102 & 0.265 & 0.083 \\
\hline $40^{\circ}$ & 0.246 & 0.081 & 0.334 & 0.032 & 0.307 & 0.066 & 0.270 & 0.069 \\
\hline $30^{\circ}$ & 0.212 & 0.091 & 0.329 & 0.033 & 0.303 & 0.047 & 0.274 & 0.051 \\
\hline $20^{\circ}$ & 0.177 & 0.089 & 0.323 & 0.031 & 0.298 & 0.037 & 0.274 & 0.054 \\
\hline $10^{\circ}$ & 0.071 & 0.099 & 0.307 & 0.026 & 0.289 & 0.028 & 0.274 & 0.063 \\
\hline $0^{\circ}$ & -0.140 & 0.214 & 0.310 & 0.020 & 0.275 & 0.022 & 0.261 & 0.066 \\
\hline$-10^{\circ}$ & 0.096 & 0.146 & 0.331 & 0.032 & 0.247 & 0.013 & 0.220 & 0.067 \\
\hline$-20^{\circ}$ & 0.253 & 0.087 & 0.355 & 0.052 & 0.240 & 0.021 & 0.125 & 0.088 \\
\hline$-30^{\circ}$ & 0.189 & 0.090 & 0.373 & 0.066 & 0.227 & 0.029 & 0.076 & 0.095 \\
\hline$-40^{\circ}$ & 0.151 & 0.099 & 0.383 & 0.087 & 0.246 & 0.033 & 0.060 & 0.097 \\
\hline$-50^{\circ}$ & 0.151 & 0.126 & 0.380 & 0.084 & 0.215 & 0.048 & 0.051 & 0.097 \\
\hline$-60^{\circ}$ & 0.188 & 0.151 & 0.372 & 0.089 & 0.266 & 0.076 & 0.047 & 0.098 \\
\hline$-70^{\circ}$ & -0.406 & 0.701 & 0.366 & 0.090 & 0.209 & 0.061 & & \\
\hline$-80^{\circ}$ & -0.378 & 0.696 & 0.356 & 0.094 & 0.210 & 0.083 & & \\
\hline Mean $^{3)}$ & 0.166 & 0.133 & 0.339 & 0.053 & 0.269 & 0.064 & 0.189 & 0.079 \\
\hline
\end{tabular}

1) Velocity is taken positive for the westward drift.

2) Standard deviations of the velocities.

3) Averaged between $60^{\circ} \mathrm{N}$ and $60^{\circ} \mathrm{S}$. 


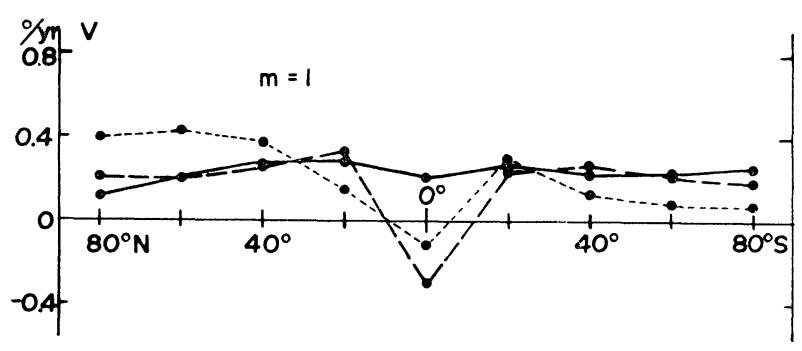

Fig. 5 Drift velocities of the harmonic number $m=1$ of the non-dipole field. The westward velocity is taken positive. The solid line is the distribution of drift rates for 1957.5, the broken line for 1942.5 and the dotted line for 1922.5 .

of the non-dipole field (Yukutake, 1962). Since the field due to the component $n=2, m=1$ has no effect on the velocity at the equator, the eastward drift at the equator would be mostly due to the harmonic $n=3, m=1$ of the main field.

As for $m=2$ and 3 of the secular variation, the distributions of the velocities are rather simple and no appreciable variation with the latitude are observable (Fig. 4). Almost all over the latitude, the westward velocity for $m=2$ exceeds $0.3^{\circ}$ year. The average between $60^{\circ} \mathrm{N}$ and $60^{\circ} \mathrm{S}$ is given as $0.34^{\circ}$ /year. For $m=3$ the velocity varies from $0.31^{\circ} /$ year to $0.22^{\circ}$ /year. For $m=4$ there exists a clear difference between the northern and the southern hemisphere. It is not certain, however, whether the difference is significant or not, because the earlier data of the secular change become less reliable for the higher harmonic components. The mean values of the drift velocities over the latitudes are calculated for the respective harmonic components and listed in Table 2.

\section{Correlation method of estimating the drift velocity}

Let $x(\phi)$ and $y(\phi)$ be the distribution of the secular variation along a specific latitude circle at two different epochs and let the westward drift be the main cause of producing the difference between the two. Then the amount of the displacement $\delta \phi$ during the period can be obtained by making a correlation between $x(\phi)$ and $y(\phi-\delta \phi)$ a maximum. This is essentially equivalent to the method employed in the analysis of the non-dipole field by Bullard et al. (1950). They determined the displacement so that the sum of the square of the difference between $x(\phi)$ and $y(\phi-\delta \phi)$ may become a minimum.

In spite of apparent simplicity of the procedure, this method requires a lot of time and labour to calculate the correlation when the values of $x(\phi)$ and $y(\phi)$ are given at finely spaced grid points. If a field is expanded in a Fourier series, the calculation of the correlation is much simplified by making use of orthogonality of trigometric functions.

We define a correlation function $\mu$ between $x$ and $y$ at a lag $\delta \phi$ as follows,

$$
\mu(\delta \phi)=\frac{\int x(\phi) y(\phi-\delta \phi) d \phi}{\left[\int\{x(\phi)\}^{2} d \phi \int\{y(\phi)\}^{2} d \phi\right]^{1 / 2}} .
$$

When $x$ and $y$ are expressed in Fourier series, 


$$
\begin{aligned}
& x=\sum_{m}\left(\alpha_{m} \cos m \phi+\beta_{m} \sin m \phi\right), \\
& y=\sum_{m}\left(\alpha_{m}{ }^{\prime} \cos m \phi+\beta_{m}{ }^{\prime} \sin m \phi\right),
\end{aligned}
$$

the function becomes,

$$
\mu(\delta \phi)=\frac{\sum_{m}\left\{\left(\alpha_{m} \alpha_{m}^{\prime}+\beta_{m} \beta_{m}^{\prime}\right) \cos (m \delta \phi)-\left(\alpha_{m} \beta_{m}^{\prime}-\alpha_{m}^{\prime} \beta_{m}\right) \sin (m \delta \phi)\right\}}{\left[\sum_{m}\left(\alpha_{m}{ }^{2}+\beta_{m}{ }^{2}\right) \sum_{m}\left(\alpha_{m}^{\prime}{ }^{2}+\beta_{m}^{\prime}{ }^{2}\right)\right]^{1 / 2}} .
$$

If a magnetic potential or an element of the field along a parallel circle at a certain epoch is represented in the form (2), and after an interval $\delta t$ in the form (3), we can determine the displacement $\delta \phi$ that maximizes the function $\mu$. Then dividing $\delta \phi$ with the interval $\delta t$, we have the mean drift rate $\frac{\delta \phi}{\delta t}$ during the period.

The procedure was applied to the secular variation of the non-dipole field. We first compared the magnetic potential for 1743 (see Yukutake and Tachinaka, 1968b) with that for 1965 (Leaton et al., 1965). The correlation functions were calculated for various latitudes and shown in Fig. 6 , where, divided by $\delta t$, the displacement $\delta \phi$ has already been transformed into the drift velocity. From the figure, the velocity that makes the function $\mu$ a maximum is obtained with an accuracy of $0.01^{\circ} /$ year. They are shown in the upper diagram of Fig. 7 by a solid line. The same procedure was repeated for the secular change in the three elements of the non-dipole field. The results for the east component and the vertical component are in good agreement with those of the magnetic potential, while the velocities for the north component were considerably scattered with respect to the

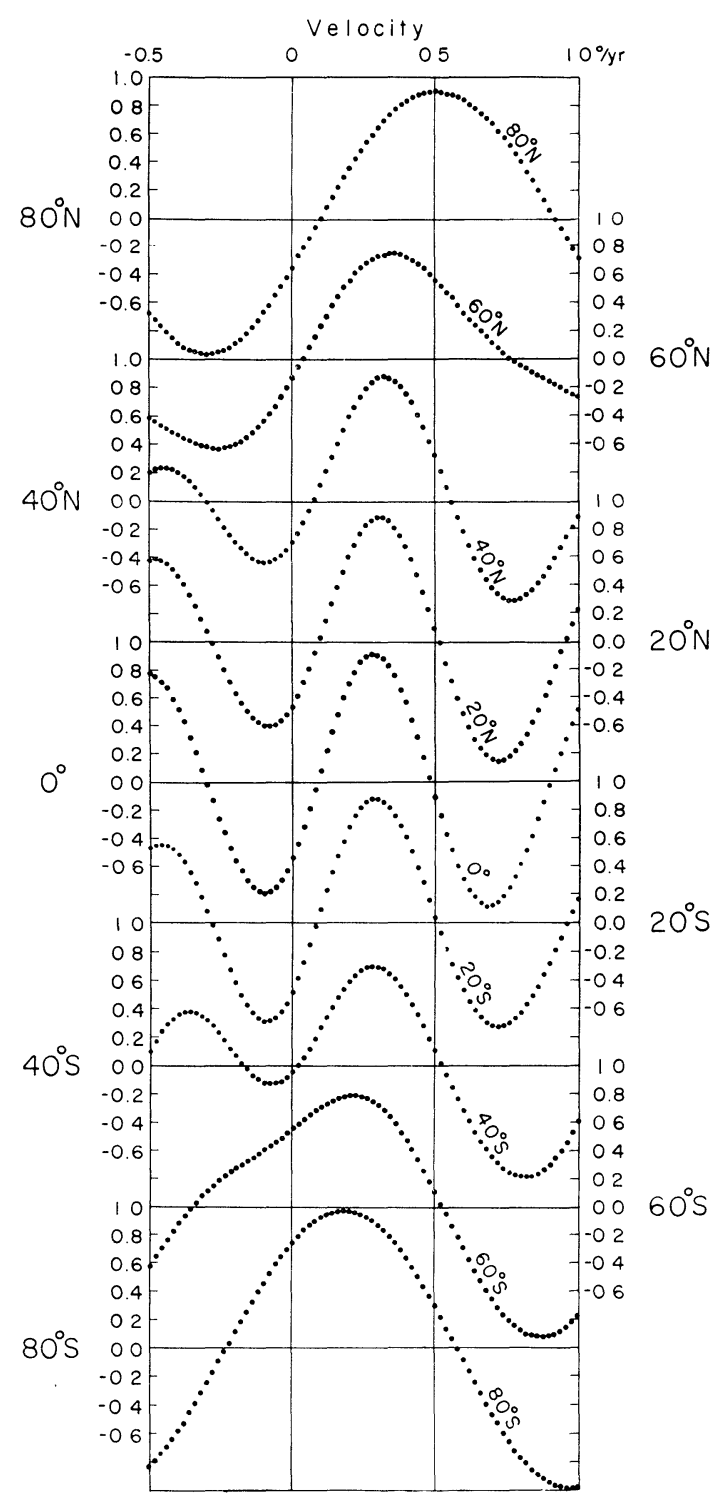

Fig. 6 The correlations $(\mu)$ of the secular change potential between the epochs 1743 and 1965 as functions of the drift velocity. 

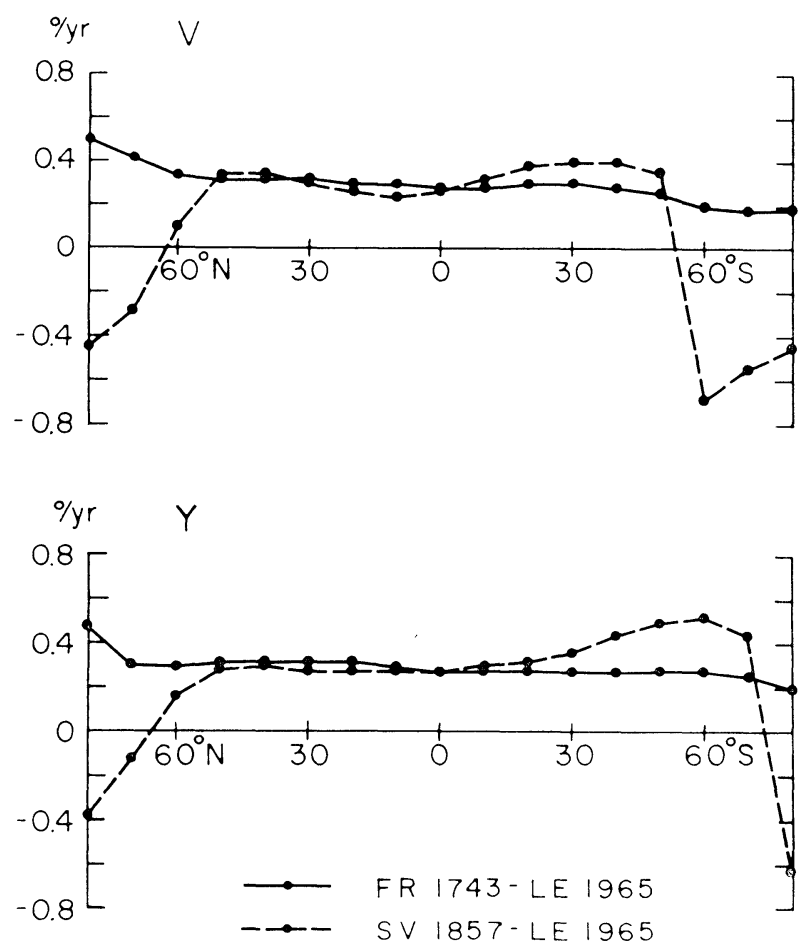

Fig. 7 Drift velocities of the secular variation in the magnetic potential $(V)$ and the east component $(Y)$ of the nondipole field. They are obtained by the correlation method. Fritsche's secular change analysis for 1743 is compared with Leaton et al.'s analysis for 1965 (solid lines) and the secular variation for 1857 with that for 1965 (broken lines).

latitude. However, we need not consider the scattered results seriously, because the spatial gradient in the east west direction is so small for the north component that a reliable velocity can hardly be determined for this component. Only the result for the east component is shown in the lower diagram of Fig. 7. The velocities obtained from the magnetic potential, the east component and the vertical component are nearly constant all over the latitudes, and no appreciable dependence on the latitude is seen, suggesting a uniform rotation of the non-dipole field. This does not necessarily contradict the eastward drift at the equator for the harmonic number $m=1$ (or $n=3, m=1$ ), because the harmonic components $n=2, m=2$ and $n=3, m=3$ dominate over the component $n=3, m=1$ and the velocities obtained at the equator are probably due to the first two harmonics. The drift velocities for the potential were averaged between $60^{\circ} \mathrm{N}$ and $60^{\circ} \mathrm{S}$ and obtained to be $0.30^{\circ}$ /year.

The same procedure has been repeated for another pair of the secular variation, i.e. the analyses for 1857 (Yukutake and Tachinaka, 1968 b) and 1965 (Leaton et al., 1965). The time interval between the analyses is about a half of the previous case. The results are shown in Fig. 7. The original data are not sufficiently reliable for the higher latitudes, that we may ignore the results for the latitudes higher than $60^{\circ}$. Then the results are highly 

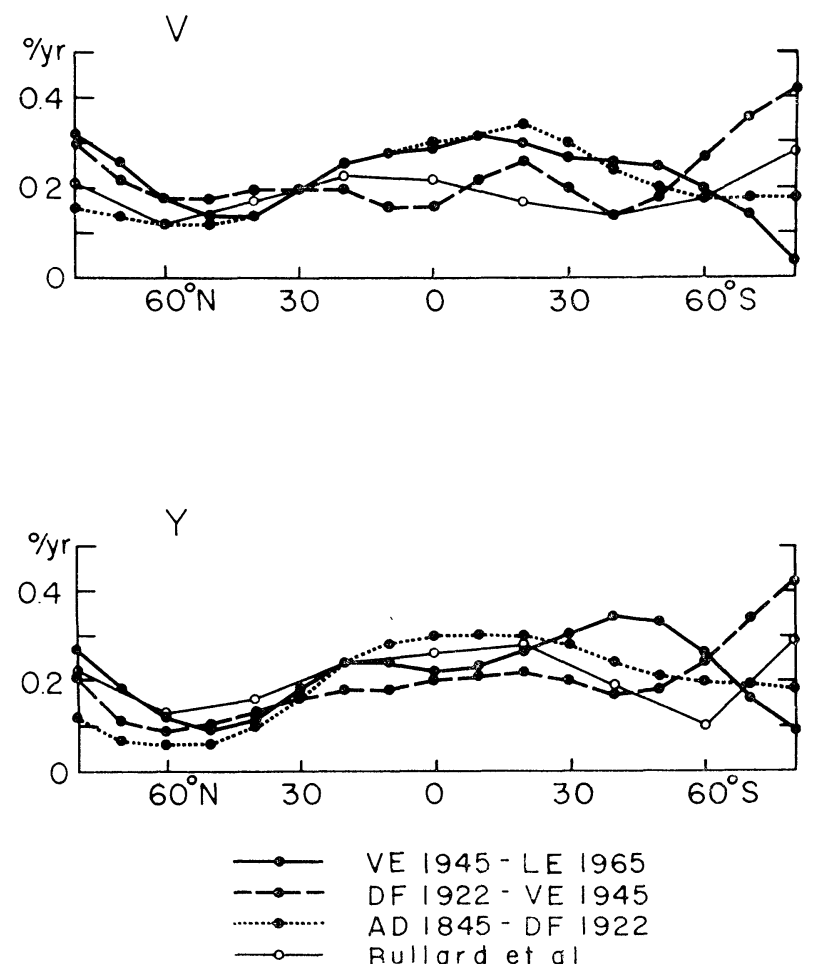

Fig. 8 Drift velocities of the non-dipole field for the magnetic potential and the east component obtained by the correlation method. Comparison is made between the non-dipole parts of the main field for various epochs: Full circles connected by the solid lines represent velocities computed from a pair of 1945 (Vestine et al.) and 1965 (Leaton et al.), the broken lines from 1922 (Dyson and Furner) and 1945 (Vestine et al.), and the dotted lines from 1845 (Adams) and 1922 (Dyson and Furner). The mean values of Bullard et al.'s results for the three components are shown in the upper diagram by open circles. In the lower diagram, Bullard et al.'s results for the east component are shown by open circles.

consistent with those for the previous combination 1743 and 1965 data, indicating that the drift velocity is approximately $0.3^{\circ}$ /year and almost independent of the latitude. Good agreement between the data of different intervals also suggests that there has been no drastic change in the drift velocity during the whole period from 1743 to 1965 .

The correlation method was also applied to the non-dipole field. From the pairs of analyses for 1845 (see Adams, 1898) and 1922 (Dyson and Furner, 1923), 1922 and 1945 (Vestine et al., 1947), and 1945 and 1965 (Leaton et al., 1965), the drift velocities are calculated and shown in Fig. 8. The upper diagram represents those of the magnetic potential and the lower ones those of the east component. The drift velocities obtained by Bullard et al. (1950) are plotted by open circles in the figure. All the results agree well with each other.

What is common to all the results for the non-dipole field is that a low velocity zone 
appears between $30^{\circ} \mathrm{N}$ and $70^{\circ} \mathrm{N}$, where the smallest velocity drops to $0.06^{\circ} /$ year. No such zone is observed for the secular variation (Fig. 7). In these latitudes there exist large regional anomalies, the Mongolian anomaly and the North American one that have been stationary over a period longer than a few hundred years (Yukutake and Tachinaka, 1968a). These anomalies seem directly responsible for reducing the drift rates in the latitudes. Similar reduction of the drift velocity may also happen when a world average is taken of the drift rates of the non-dipole field. This seems to be the main reason why the nondipole field gives the low mean velocity $0.2^{\circ}$ /year, despite that the velocity of the non-dipole field is nearly the same near the equator as that of the secular variation, $0.3^{\circ} /$ year.

\section{Discussion about the drift velocity}

If standing anomalies do not change their intensity, the drift velocity obtained from the geomagnetic secular variation is completely free from the influence of those anomalies. However, when there is even a slight change in the intensity, it will have certain effect on the estimated velocity. How far such a kind of error is involved in the results depends on the methods employed. Among the three methods presented in this paper, the first one, the phase angle method for individual spherical harmonic components will be most largely influenced. If a standing anomaly that change its intensity had such a distribution as might be expressed by a single harmonic component, the obtained velocity for the component would be considerably different from the actual drift velocity. Such an effect would not be so dominated in the case of a Fourier component method as in the phase angle method for the spherical harmonics, and even more diluted in the correlation method, where a rigid rotation of the whole distribution of the secular variation is assumed along a parallel. The velocity is mainly determined by the drifting field with the largest intensity in the last case.

On account of the reason described above, the possibility that the dispersive velocities obtained for the individual spherical harmonic components involve the effect of the standing anomaly cannot be excluded. However, the second method based on the Fourier expansion of the secular change also suggests that the velocity is dependent on the harmonic number. Even if the extremely low velocity in the equatorial region is excepted, the velocity for $m=1$ is smaller than $0.2^{\circ}$ year, while that for $m=2$ is everywhere larger than $0.3^{\circ} /$ year. The difference seems significant beyond various kind of errors involved. As for the harmonic number $m=3$, no clear difference is noticed from $m=2$. It may be concluded that there exists a dispersive relationship in the drift velocity, at least between the harmonics $m=1$ and $m=2$ of a Fourier series.

From sections 3 and 4, no remarkable latitude dependence of the drift velocity is noted except for the equatorial region, suggesting that the westward drift is more like a rigid rotation for respective harmonic component. An equatorial eastward drift or oscillation of the field with the harmonic component $m=1$ has been revealed in section 3 . This is probably associated with the queer behaviour of the spherical harmonic component $n=3, m=1$. It is not necessarily unreasonable to suppose a peculiar behaviour in the equatorial region, where the Coriolis force has small effect on the lateral motion of fluid near the surface of 
the earth's core. However, more detailed analysis seems necessary to discuss the hydrodynamic process in the core in relation to the equatorial counter drift.

\section{References}

Adam, N.V., N.P. Ben'kova, V.P. Orlov and L.O. Tyurmina, Western drift of the geomagnetic field, Geomag. Aeron., 4, 434-441 (English), 1964.

Adams, W.G., An account of the late Professor John Couch Adams's determination of the Gaussian magnetic constants, Brit. Ass. Adv. Sci., Bristol Meeting 1898, Internat. Conf. on Terr. Mag. and Atm. Elect., 22-49, 1898.

Bullard, E.C., C. Freedman, H. Gellman and J. Nixon, The westward drift of the earth's magnetic field, Phil. Trans. Roy. Soc. London, A, 243, 67-92, 1950.

Dyson, E. and H. Furner, The earth's magnetic potential, Mon. Not. Roy. Astr. Soc. London, Geophys. Suppl., 1, 76-88, 1923.

Hide, R., Free hydromagnetic oscillations of the earth's core and the theory of the geomagnetic secular variation, Phil. Trans. Roy. Soc. London, A, 259, 615-650, 1966.

Leaton, B.R., S.R.C. Malin and M.J. Evans, An analytical representation of the estimated geomagnetic field and its secular change for the epoch 1965.0, Jour. Geomag. Geoelect., 17, 187-194, 1965.

Nagata, T., The main aspects of geomagnetic secular variation-westward drift and non-drifting component, Proc. Benedum Earth Magnetism Symp., 39-55, 1962.

Vestine, E.H., L. Laporte, I. Lange and W.E. Scott, The geomagnetic field, its description and analysis, Carnegie Inst. Wash. Publ., 580, 1-390, 1947.

Whitham, K., The relationships between the secular change and the non-dipole fields, Canad. Jour. Phys., 36, 1372-1396, 1958.

Yukutake, T., The westward drift of the magnetic field of the earth, Bull. Earthq. Res. Inst., 40, 1-65, 1962.

Yukutake, T., The westward drift of the earth's magnetic field in historic times, Jour. Geomag. Geoelect., 19, 103-116, 1967.

Yukutake, T. and H. Tachinaka, The non-dipole part of the earth's magnetic field, Bull. Earthq. Res. Inst., 46, $1968 \mathrm{a}$, in press.

Yukutake, T. and H. Tachinaka, The westward drift of the geomagnetic secular variation, Bull. Earthq. Res. Inst., 46, $1968 \mathrm{~b}$, in press. 